\title{
Chapter 8 \\ The Virtuous Physician and Antimicrobial Prescribing Policy and Practice
}

\author{
Justin Oakley
}

\begin{abstract}
In this chapter, I outline some key patient-centred medical virtues and several community-centred medical virtues, and I consider what sorts of antimicrobial prescribing decisions such virtues would lead physicians to make. I argue that practically-intelligent virtuous physicians should also have an awareness of the sorts of cognitive biases that are especially likely to distort their antimicrobial prescribing decisions, and I urge physicians to develop ways of avoiding or counteracting such biases. Further, I argue that effectively addressing the impact of these biases and other countervailing factors that inhibit virtuous prescribing practices is the responsibility not only of individual physicians, but also of institutions and regulators. I outline some strategies that individual physicians, institutions, and healthcare policymakers could develop to help physicians hit the targets of those patient-centred and community-centred medical virtues, and to thereby play their part in redressing the problems of antimicrobial resistance.
\end{abstract}

Keywords Medical virtue $\cdot$ Practical intelligence $\cdot$ Justice $\cdot$ Antibiotic overprescribing $\cdot$ Cognitive bias

The detrimental health impact of antimicrobial resistance raises significant questions about physicians' antimicrobial prescribing decisions, so it is important to investigate what sorts of prescribing decisions about antimicrobials would be made by a virtuous physician in various contexts. Antimicrobial resistance has become a

\footnotetext{
An earlier version of this chapter was presented at the 2018 Effective Altruism Global Conference, Melbourne, where I received some helpful comments on this chapter. I also received some useful feedback on these ideas in medical ethics seminars at Monash University, and I thank students at these seminars for this. I am very grateful to Zeb Jamrozik for his resourcefulness and his excellent suggestions about relevant sources, and I wish to thank Michael Selgelid and Zeb for their invaluable advice with this chapter.
}

J. Oakley $(\bowtie)$

Monash Bioethics Centre, Monash University, Melbourne, VIC, Australia

e-mail: justin.oakley@monash.edu 
major global health concern, as the resistance of disease-causing microorganisms to antimicrobials can completely undermine the effectiveness of many antibiotics and other antimicrobials that are commonly used to treat a variety of diseases, and to lower the risks of post-surgical infections. The dwindling effectiveness of many antimicrobials has been implicated in a growing number of patient deaths worldwide, often from conditions such as respiratory and wound infections which had previously been responsive to antimicrobial treatments. Addressing what sorts of antimicrobial prescribing decisions a virtuous physician would make seems especially challenging for virtue ethics approaches to medical practice. For such approaches typically evaluate physician decision-making by reference to patientcentred role virtues like medical beneficence, medical courage, and trustworthiness, but tend to say little about broader medical virtues that help physicians act in the best health interests of the community, which is a crucial consideration in ethically justifiable clinician decision-making regarding antimicrobials.

In this chapter, I discuss some key patient-centred medical virtues and several candidate community-centred medical virtues, and I consider what sorts of antimicrobial prescribing decisions such virtues would lead physicians to make. I draw out the moral significance of these community-centred medical virtues by examining certain cases where prescribing an antimicrobial seems likely to be in the best interests of the patient in question, but seems unlikely to be in the best health interests of the community overall. In doing so, I consider some analogies with other sorts of cases involving seemingly divergent virtue directives, such as those involving psychiatrists breaching patient confidentiality to protect third parties from harm. Also, I argue that practically-intelligent virtuous physicians should have an awareness of the sorts of cognitive biases that are especially likely to distort their antimicrobial prescribing decisions, and I urge physicians to develop ways of avoiding or counteracting such biases. Further, effectively addressing the impact of these biases and other countervailing factors that inhibit virtuous prescribing practices is, I argue, the responsibility not only of individual physicians, but also of institutions and regulators. Therefore, I also consider the prospects of certain institutional and regulatory initiatives which aim to reduce antimicrobial over-prescribing by highlighting and undermining such biases in clinical practice.

\subsection{Antimicrobial Resistance and Virtue Ethics}

Rising global concerns about antimicrobial resistance has prompted many national and international agencies, professional medical associations, and policymakers to investigate what sorts of factors are contributing to this problem, and to the resulting significant increases in morbidity and mortality rates of certain diseases in many countries around the world. The accelerating resistance of microorganisms to antibiotics and other antimicrobial agents commonly used in medical treatment has substantially reduced - and at times entirely removed - effective microbial treatment options for some patients with serious conditions such as pneumonia, 
tuberculosis, or septicaemia, and a number of those patients have subsequently died. Further, antibiotic-resistant 'superbugs' like methicillin-resistant staphylococcus aureus (MRSA) have been found in significant quantities in some of the world's major hospitals, and many patients worldwide have died from hospital-acquired MRSA infections. ${ }^{1}$ Antimicrobial resistance is a major problem in high-income countries and also in low- and middle-income countries. For example, China has the world's highest prevalence of MRSA, and this bacterium is associated with higher mortality rates for hospitalised patients there. ${ }^{2}$ Also, the prevalence of multi-drug resistant tuberculosis is relatively high in countries such as India and Russia - it has recently been predicted that $12.4 \%$ of all cases of TB will soon be multi-drug resistant in India, and that $32.5 \%$ of all cases of TB will soon be multi-drug resistant in Russia. ${ }^{3}$ It has also been reported that over $50 \%$ of microorganisms involved in hospital-acquired infections in Greece seem to be resistant to all available antibiotics. ${ }^{4}$ Effective antimicrobial treatments have therefore become a somewhat scarce resource in clinical practice in a number of contexts, and many countries have now introduced new government regulations and clinical guidelines aimed at encouraging more responsible use and stewardship of antimicrobials. While antibiotics are one among several antimicrobial agents, I will focus here on the prescription of antibiotics, as inappropriate antibiotic prescribing appears to be a major contributor to antimicrobial resistance, which is why many of the efforts to improve antimicrobial prescribing practices are directed at antibiotic prescribing in particular.

This accelerating growth in antimicrobial resistance is due to a range of factors, including widespread farming practices, patient misuse of antimicrobials, and inappropriate antimicrobial prescribing practices by physicians. These contributing factors are now being targeted by a range of initiatives from government agencies, national and international medical and health care organisations, and at the level of hospital management. Thus, the WHO recently modified its longstanding Essential Medicines List by introducing recommendations that prioritise the use of some antibiotics over others in certain contexts - for example, the list recommends that amoxicillin be made widely available to treat common infections, such as pneumonia, but that other antibiotics should be reserved for use as a last resort when a life-threatening infection has failed to respond to other antibiotics. ${ }^{5}$ And the clinical practice guidelines about antibiotic prescribing in Australia's Therapeutic Guidelines: Antibiotic also encourage the responsible use by clinicians of antibiotics in treating a wide variety of conditions, and thus discourage the over-prescribing of antibiotics. ${ }^{6}$ However, questions have arisen about the effectiveness of clinical

\footnotetext{
${ }^{1}$ Staphylococcus aureus already exist in (e.g.) our respiratory tract, and some of those bacteria will already be antimicrobial-resistant, but antibiotic treatment allows those antimicrobial resistant S. aureus to potentially multiply.
}

${ }^{2}$ See Dan Cui et al. (2017), and Zhenjiang Yao et al. (2015).

${ }^{3}$ Aditya Sharma et al. (2017).

${ }^{4}$ Stavros Saripanidas (2016).

${ }^{5}$ See http://www.who.int/mediacentre/news/releases/2017/essential-medicines-list/en/.

${ }^{6}$ Antibiotic Expert Groups (2019). 
guidelines aimed at improving antibiotic prescribing. For example, a recent Australian study concluded that "Antibiotics are prescribed for ARIs [acute respiratory infections] at rates 4-9 times as high as those recommended by clinical guidelines. The potential for reducing rates of antibiotic prescription and to thereby reduce rates of antibiotic-related harms, particularly bacterial resistance, is there-

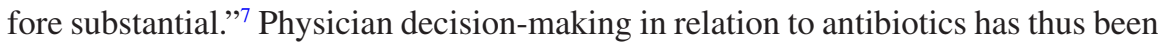
identified as a major contributor to the problem of antimicrobial resistance, ${ }^{8}$ and so this remains a promising context on which to focus in developing effective ways of redressing this problem. In this chapter, therefore, I concentrate on the antimicrobial prescribing behaviour of physicians, and on what would plausibly be required of a virtuous physician in regard to antimicrobial prescribing.

The emphasis in virtue ethics on the character and moral psychology of virtuous agents have led some to regard this approach as excessively individualistic, and so it might be wondered whether virtue ethics is capable of providing useful guidance on addressing antimicrobial resistance, which is a complex multi-dimensional problem on an international scale. However, as noted above, physician prescribing behaviour is a major contributing factor to antimicrobial resistance, and so to this extent, focusing on what decisions a virtuous physician would make in relation to the prescription of antimicrobials would seem to offer a promising line of response to this problem. Further, recent empirically-informed accounts of the Aristotelian idea of practical intelligence (phronesis) emphasise the importance of agents being aware of, and having strategies to counteract, common decision-making biases that can divert their virtuous dispositions from hitting the target of the relevant virtue. ${ }^{9}$ Physicians are evidently not immune from such biases, ${ }^{10}$ and these biases may well contribute to physicians' inappropriate antimicrobial prescribing in certain sorts of cases. ${ }^{11}$ So applying recent empirically-informed accounts of virtues and practical intelligence to physicians' roles and to their antimicrobial prescribing behaviour would seem to be a useful way of approaching the problems of antimicrobial resistance.

Generally speaking, virtue ethics evaluates actions by asking, 'what sort of person would do a thing like that?' For example, we can ask whether an action was generous or mean-spirited, courageous or cowardly, and we can examine whether this is the sort of thing which a kind person or a just person would do in the circumstances. Virtue ethics can therefore draw upon such considerations to provide the elements of a criterion of right action. A virtue ethics criterion of right action can be

\footnotetext{
${ }^{7}$ Amanda R. McCullough et al. (2017).

${ }^{8}$ See e.g. the US Centers for Disease Control and Prevention statement, 'About Antimicrobial Resistance': "The use of antibiotics is the single most important factor leading to antibiotic resistance around the world. Simply using antibiotics creates resistance. These drugs should only be used to manage infections." https://www.cdc.gov/drugresistance/about.html. [accessed 1 November 2018].

${ }^{9}$ See Justin Oakley $(2018 \mathrm{a}, \mathrm{b})$.

${ }^{10}$ See Blumenthal-Barby and Krieger (2015) and Saposnik et al. (2016).

${ }^{11}$ See e.g. Jeffrey et al. (2014).
} 
stated initially in broad terms as holding that: An action is right if, and only if, it is what an agent with a virtuous character would do in the circumstances. ${ }^{12}$ Virtue ethicists have also applied such an appeal to exemplars in developing accounts of right action in the context of various professional roles, such as medical and legal practice. An influential version of these accounts highlights links between the proper goal/s of the profession in question - such as health and justice for medicine and law, respectively - and an Aristotelian conception of human flourishing, or eudaimonia. The proper goal/s of a profession can, in turn, provide the basis for an account of the role virtues for that profession. Thus in the case of medical practice, the role virtues for doctors can be understood as those character traits that enable doctors to serve the goal of health. These traits have been thought to include patientcentred dispositions such as medical beneficence, medical courage, and trustworthiness, along with community-centred dispositions such as justice. ${ }^{13}$

\subsection{Medical Virtues and Antimicrobial Prescribing}

We saw in the previous section that inappropriate prescribing decisions by physicians in relation to antibiotics is highlighted as one of the most important contributing factors to antimicrobial resistance and its resulting problems. Physicians in many countries are evidently over-prescribing antibiotics, and for a variety of conditions. For example, a recent study by Katherine Fleming-Dutra and colleagues found that during 2010-2011 physicians in US clinics prescribed antibiotics for acute respiratory infections at double the rates that were clinically appropriate for such infections - in other words, around half of those antibiotic prescriptions were clinically inappropriate. ${ }^{14}$ Also, the 2018 national survey of antimicrobial prescribing practices in Australian hospitals found that $21.4 \%$ of antimicrobials prescribed during that year were classified as inappropriate prescriptions. ${ }^{15}$ And a recent study using survey data from 2010-2015 on antibiotic prescribing rates in general practice found that Australian GPs are prescribing antibiotics for acute respiratory infections at rates up to nine times higher than those recommended by the national Therapeutic Guidelines: Antibiotic. These researchers found, for example, that "Despite the recommendation in these guidelines against prescribing antibiotics for bronchitis, general practitioners were estimated to have prescribed antibiotics for patients with bronchitis in $85 \%$ of cases during 2010-2015". ${ }^{16}$ A subsequent interview with study co-author Chris Del Mar reported that he felt "doctors were acting out of a misplaced sense of caution, as many conditions requiring treatment with antibiotics

\footnotetext{
${ }^{12}$ Rosalind Hursthouse (1999).

${ }^{13}$ See e.g. Justin Oakley and Dean Cocking (2001).

${ }^{14}$ Katherine E. Fleming-Dutra et al. (2016).

${ }^{15}$ Australian Commission on Safety and Quality in Health Care (2020).

${ }^{16}$ McCullough et al. (2017), op. cit., p. 68.
} 
share similar symptoms to those that do not.... doctors needed to stop considering a prescription for antibiotics as their first port of call. 'The idea that we have as GPs, that writing a prescription for antibiotics just in case, is probably not so effective..., That in fact not using antibiotics at all is actually safe, you don't end up with missed cases of meningitis and Lemierre's disease - all sorts of really nasty things.."'17

Given that patients frequently request antibiotics for acute respiratory infections, and are usually keen to get better sooner, it is perhaps not surprising that there is a tendency among physicians in many countries to over-prescribe antibiotics for such infections in responding to such requests. But while yielding to patient pressure results in physicians over-prescribing antibiotics, there is also evidence that antibiotics are prescribed inappropriately because physicians overestimate patients' expectations of receiving these drugs. Thus, in a 1997 study of antibiotic prescribing decisions by UK General Practitioners, Macfarlane and colleagues found that "Non-clinical factors influence the decision to prescribe antibiotics for nearly a half of those receiving one. Patient pressure was cited most frequently... and identified by the Audit Commission as an important reason for the excess use of antibiotics in the community. Pressure from patients to prescribe antibiotics, particularly for respiratory symptoms, has been identified as the commonest reason for doctors' discomfort with prescribing decisions. General practitioners can, however, overestimate patients' expectations. A quarter of our patients received antibiotics when they stated that before the consultation they had not wanted antibiotics". ${ }^{18}$ A survey of 544 patients of inner London general practitioners found further evidence that physicians are overly influenced by their perceptions of patients' hopes and expectations of receiving a medication prescription, and those perceptions frequently seemed to be inaccurate: "A third [31\%] of the prescriptions [which were sometimes for antibiotics] written in this study were either not indicated or not hoped for, with 3\% being neither indicated nor hoped for.... The writing of nonindicated prescriptions was primarily associated with the doctor's sense of feeling pressurised [by the patient to write a prescription]". ${ }^{19}$ This survey also found that "In a fifth [22\%] of consultations in which a prescription was written, the prescription was not strictly indicated on purely medical grounds". ${ }^{20}$ Overall, the strongest influence on a physician's decision to prescribe a medication was found in this study to be the physician's perceptions of their patients' hopes and expectations about receiving that medication.

\footnotetext{
${ }^{17}$ Angus Randall (2017).

${ }^{18}$ John Macfarlane et al. (1997), p. 1213. There is also evidence that UK General Practitioners have overestimated patients' expectations of receiving a prescription, not only in cases involving antibiotics, but also with other sorts of medication: see Nicky Britten (1995), p. 1084: "When general practitioners are surveyed they describe high levels of demand, but objective evidence consistently suggests that doctors overestimate patients' expectations. Reanalysis of published data shows that about a fifth of patients leave general practice consultations with prescriptions they did not expect."

${ }^{19}$ Nicky Britten and Obioha Ukoumunne (1997), p. 1509.

${ }^{20}$ Ibid, p. 1510.
} 
In some of the above types of cases where physicians prescribe antibiotics inappropriately, they may well be acting from a beneficent motivation to serve the best interests of their patient in some way - particularly where, as mentioned above, physicians prescribe an antibiotic "just in case" the patient has a serious, albeit rare, type of infection where antibiotic treatment at that stage would likely benefit the patient in the longer-term. However, physicians are frequently in a position to accurately diagnose that a patient has the less serious condition of acute bronchitis, and to recognise that it is in the long-term best interests of such a patient not to be prescribed an antibiotic now. ${ }^{21}$ Where physicians prescribing antibiotics are acting in misguided ways from motives of beneficence towards their patients, these cases can be usefully characterised as involving a failure of such motives to hit the target of the patient-centred virtue of medical beneficence. ${ }^{22}$ Cognitive biases seem to be an important factor in explaining why physicians' beneficent motives can fail to hit the target of the virtue of medical beneficence in their prescribing decisions. An illuminating recent study of Australian GP trainees (registrars) describes their decisionmaking processes in deciding whether or not to prescribe an antibiotic for the patient. A number of these registrars explained that, while they realised prescribing an antibiotic was often not in the patient's best interests, and that it was important to avoid antibiotic over-prescribing, the registrars indicated how they rationalised prescribing an antibiotic in certain cases - for instance, telling themselves that it was in the patient's best interests to receive an antibiotic. The study found that: "Many registrars were concerned about patient safety and avoiding subsequent hospital presentations [for example, one registrar said]: 'I tend to probably cover things a bit more because...if something goes wrong I want to make sure that the patient is going to be safe.' (Registrar 9)" ${ }^{23}$ In certain cases this seems to be an overly cautious approach, which may indicate that a registrar is unduly influenced by the wellknown 'framing effect', whereby possible losses loom larger to the agent than do possible gains. ${ }^{24}$ The researchers also mentioned that: "Barriers to evidence-based prescribing included role-modelling outdated practices, or setting a precedent of

\footnotetext{
${ }^{21}$ See McCullough et al., op. cit. For a useful systematic review of studies on how individuals treated with an antibiotic for respiratory and urinary infections can subsequently develop a higher level of bacterial resistance to that antibiotic for several months, see Céire Costelloe et al. (2010). ${ }^{22}$ Christine Swanton emphasizes the importance of virtues 'hitting the target' of the contextuallyrelevant virtue, and she discusses various examples of candidate virtues that fail to hit their target (see Christine Swanton 2003). Similarly, Dean Cocking and I have argued that each virtue involves being guided by a 'regulative ideal': "To say that an agent has a regulative ideal is to say that they have internalised a certain conception of correctness or excellence, in such a way that they are able to adjust their motivation and conduct so that it conforms - or at least does not conflict - with that standard" (Oakley and Cocking 2001, op. cit., p. 25).

${ }^{23}$ Anthea Dallas et al. (2014), p. e564. While antibiotic over-prescribing seemed due in part to certain cognitive biases in the registrars, "many registrars described a dissonance between their attitudes to guidelines and their prescribing behaviours, producing dissatisfaction with their own prescribing...: 'There's probably been times where I've given them the script and kind of felt a bit disappointed in myself afterwards.'(Registrar 14)” p. e563.

${ }^{24}$ See Amos Tversky and Daniel Kahneman (1981).
} 
prescribing that created patient expectations and pressure on the registrar: ' $I$ do know one supervisor in particular will give his patients antibiotics even for something that sounds very viral, and therefore when I see his patients, Ifeel I'm expected to do that as well, because his patients have been seeing him for many years. So they expect it too, so I'm definitely more likely to give his patients antibiotics even when I don't think it's justified." (Registrar 10) ${ }^{25}$ This sort of approach may manifest a form of 'authority bias', whereby the registrar shows undue deference towards the antibiotic prescribing practices of his or her clinical supervisor. ${ }^{26}$

According to some influential recent empirically-informed accounts of virtue and virtue ethics, acting virtuously requires (among other things) agents to employ certain deliberative strategies to counteract common decision-making biases and other countervailing factors which can impede virtuous actions. These accounts develop a comprehensive conception of virtuous character-traits, which include an awareness of situational factors that conduce to or inhibit virtuous behaviour. ${ }^{27}$ The misguided beneficent efforts of those registrars who were (or could reasonably have been) aware that prescribing an antibiotic was not in their patient's best interests can be helpfully understood as failures to exercise the central Aristotelian virtue of phronesis, or practical intelligence, in this context. ${ }^{28}$ Broadly speaking, phronesis is an overarching normative disposition that regulates the more specific dispositions involved in particular virtues, to enable their more context-sensitive expression. Developing such practical intelligence can help well-motivated agents to avoid two potential deficiencies that can undermine their attempts to hit the target of the relevant virtue - i.e. moral ineptitude, and failures of meticulousness. Moral ineptitude is shown by agents who are well-intentioned but lack sufficient practical know-how or emotional intelligence to succeed in bringing about the good which they intend to bring about. Unmeticulous agents have the relevant practical know-how and emotional intelligence, but their efforts fall short as they do not apply strategies to circumvent decision-making biases (and similar countervailing factors) that are prevalent in the context. ${ }^{29}$ Thus, the registrars who prescribed antibiotics against their patients' longer-term best interests failed to exercise the virtue of practical intelligence here, because the registrars were not sufficiently meticulous in preventing cognitive biases and other countervailing factors from undermining their efforts to make prescribing decisions in their patients' best interests. ${ }^{30}$

\footnotetext{
${ }^{25}$ Dallas et al., p. e565.

${ }^{26}$ The phenomenon of 'authority bias' was famously observed in Stanley Milgram's experiments on obedience to authority. See Stanley Milgram (2010).

${ }^{27}$ See e.g. Daniel C. Russell (2009), e.g. p. 140; Nancy E. Snow (2009), p. 563; and Nancy E. Snow (2010), especially her pp. 34-7 discussion of how people can effectively confront and combat any prejudices they might hold. These conceptions of virtue and virtue ethics have been developed in response to 'situationist' critiques of earlier accounts of virtue ethics.

${ }^{28}$ Aristotle (1980); and Russell (2009), op. cit.

${ }^{29}$ For further details about these executive failings, and their relevance to practical intelligence and medical virtues, see Oakley (2018b), op. cit. See also Oakley (2018a), op. cit.

${ }^{30}$ See also Daniel Russell's helpful discussion of how agents may ascertain the specific ends of virtuous actions, in: Daniel C. Russell (2015).
} 
Indeed, there is evidence of other cognitive biases diverting physicians' prescribing decisions from patients' best interests. For example, a recent US study found that as the number of prescribing decisions made by each primary care physician increased through the morning and into the afternoon, each physician became more likely to issue an antibiotic prescription that was not in the patient's best interests. This study suggests that good prescribing behaviour can be undermined by a form of 'decision fatigue' (a phenomenon seen in many other contexts), which seemed to lead the physicians to become less able to resist providing inappropriate antibiotic prescriptions. ${ }^{31}$ So, developing ways of effectively addressing these (and potentially other) cognitive biases that appear to be contributing to physicians prescribing antibiotics in cases where this is likely to be contrary to the patient's long-term best interests, would seem a promising strategy supported by an empirically-informed virtue ethics approach (and perhaps also by certain other ethical approaches) to the problems of antimicrobial resistance.

\subsection{Community-Centred Medical Virtues and Antimicrobial Prescribing Practice and Policy}

Virtue ethics approaches to medical practice have tended to focus predominantly on patient-centred virtues, such as medical beneficence and medical courage, which help an individual doctor serve well the best interests of their patients. But while it is important that these virtues correct any physician tendencies to make unreflective assumptions about what prescribing decisions are best for their patients, patientcentred virtues are not the only role virtues which are relevant to ethically justifiable prescribing behaviour and the problem of antimicrobial resistance. For the antimicrobial prescribing decisions by physicians have also contributed to broader community harms, such as the diminishing effectiveness of antibiotic treatments for other patients, and the scarcity of more expensive, last resort, antibiotics due to their increasing use as first-line treatments (and which have become unaffordable to patients in some countries). So, ethically responsible antibiotic prescribing practices by physicians must also take into account the broader effects that their prescribing decisions are likely to have on the community. The virtuous antimicrobial prescriber thus needs to take account of a wider moral universe, beyond that of the best interests of their own patients. They would therefore be guided in their antimicrobial prescribing decisions by community-centred medical virtues, such as justice and a readiness to serve the broader community, along with patient-centred virtues such as medical beneficence. The virtue of justice requires physicians to allocate fairly the medical resources under their control. ${ }^{32}$ In Aristotelian terms, allocating

\footnotetext{
${ }^{31}$ Linder et al. (2014), op. cit.

${ }^{32}$ For a useful discussion of how medical benevolence should be constrained by the virtue of justice, see Roger Crisp (2015).
} 
medical resources fairly (particularly when medical resources are scarce) can be understood as making allocation decisions in such a way as to provide each person who is affected by the decision an equal chance of developing and exercising their capabilities to live a flourishing human life. These capabilities include those elaborated in the Nicomachean Ethics, such as being able to understand the world, to engage in practical reasoning about our lives, and to form personal relationships with others. ${ }^{33}$ The readiness to serve others is another community-centred medical virtue, which requires physicians to make their services broadly available to the community, and so (for example) to avoid picking and choosing their patients according to the physician's personal preferences. This virtue is plausibly understood as applying to physicians qua professionals, who ought to provide this readiness to serve the community in return for being granted a monopoly of expertise over the provision of key goods - i.e. those that serve patients' health. ${ }^{34}$

In the context of antimicrobial prescribing, the virtue of justice requires physicians to consider whether prescribing antibiotics for patients in certain circumstances is likely to detrimentally affect the availability of effective antibiotics for other patients, even if there are grounds for believing that an antibiotic prescription is likely to be of some benefit to the former patients. While it is plausible to believe that physicians often serve the health of the community best by making antibiotic prescribing decisions that are in the best interests of their own patients, there will be cases where these two goals come into conflict - because the community's interest in constraining antimicrobial resistance can sometimes be served best by the physician refraining from providing an antibiotic which may be of some benefit to their patient. Where the likely benefit to the patient in such conflict cases is only marginal, the virtue of justice will require a physician not to prescribe antibiotic treatment to their own patient. Of course, it is possible that justice could also sometimes require withholding from a patient an antibiotic that is likely to be of greater than marginal benefit - for instance, where there is only a single last-resort antibiotic available, and one patient is likely to derive much greater benefit from receiving this antibiotic than is another patient, then justice could arguably require that the first patient be provided with this antibiotic. (This is analogous to situations where justice can plausibly require the allocation of the only available ICU bed to a patient who is likely to benefit more from this resource than will another patient, even if this second patient may suffer considerable harm as a consequence.) In what follows, I will concentrate on the first kind of conflict cases.

Suppose a child presents to their physician with acute otitis media. Prescribing an antibiotic in such cases would appear likely to confer marginal benefits for the child - but as Collignon explains, "with an absolute reduction in pain in only $5 \%$. Most cases resolve spontaneously. Seventeen children must be treated to prevent

\footnotetext{
${ }^{33}$ See the capabilities approach developed by Martha C. Nussbaum (2006). Nussbaum draws on Aristotle's view that "the form of government is best in which every man, whoever he is, can act best and live happily" (Politics 1324a23-5). See also Justin Oakley (1994); and Millar, M. (2020).

${ }^{34}$ This community-centred virtue becomes especially relevant in discussions of ethically justifiable limits on conscientious objection by physicians.
} 
one child having some pain after two days. Antibiotics have no effect on hearing problems or other complications" ${ }^{35}$ In these sorts of cases, despite the possible minor benefits of the child receiving an antibiotic, the detrimental impact which such patterns of antibiotic prescribing have on antimicrobial resistance in the broader community suggests that the virtue of justice would require physicians not to prescribe antibiotics in such cases, and to provide the child with other medication, to relieve their symptoms. The dictates of justice in these sorts of cases can be compared with what justice would plausibly require of psychiatrists deliberating about whether to breach patient confidentiality to protect third parties from harm. Where a patient confides to his or her psychiatrist a credible threat to significantly harm a third party, and the psychiatrist is able to take steps to see that the third party is warned about this threat, the virtue of justice plausibly requires the psychiatrist to take such steps in the interests of this third party. ${ }^{36}$ While maintaining absolute patient confidentiality might sometimes be in the best interests of the patient involved, and so might be thought consistent with the virtue of medical beneficence, the overarching virtue of justice requires that confidentiality be breached here. (Indeed, in many such cases this course of action will not be contrary to the virtue of medical beneficence - for instance, where confiding such a threat is actually a 'cry for help' from the patient - even if beneficence provides some grounds for maintaining confidentiality here).

But while the virtue of justice and its concerns for the broader interests of the community can in certain circumstances require physicians to refrain from providing a patient with an antibiotic that is likely to benefit that patient, there is evidence that physicians relegate these broader interests to the periphery of their antibiotic prescribing decision-making. A 2002 US survey of 400 generalist physicians and 429 infectious diseases specialists found that: "The risk of contributing to the problem of antibiotic resistance was ranked lowest, overall and by generalists, and second lowest by ID specialists" ${ }^{37}$ The researchers concluded that " ....neither generalists nor infectious disease specialists emphasize the relative societal risks of antimicrobial drug selection in their treatment decisions for patients with community-acquired pneumonia. Instead, they emphasize providing the newest and best treatments for each individual patient even though this approach may not be supported by current guidelines or public health policy". ${ }^{38}$ One explanation of the insufficient weight given by these doctors to the risk of generating antimicrobial resistance was an overconfidence that their own antibiotic prescribing decisions were unlikely to negatively impact on antimicrobial resistance. This can be seen as an example of the cognitive bias known as 'the overconfidence effect', whereby agents have greater confidence in their judgements than is warranted by the evidence. There is much

\footnotetext{
${ }^{35}$ Peter J. Collignon (2002), p. 328.

${ }^{36}$ See, for example, the much-discussed 1969 case of Tatiana Tarasoff, as described in Marilyn McMahon (1992), pp. 12-16.

${ }^{37}$ Joshua P. Metlay et al. (2002), p. 90.

${ }^{38}$ Ibid., p. 93.
} 
evidence that physician decision-making can be distorted by an overconfidence bias, which is one of the most frequently studied biases in medical decisionmaking. ${ }^{39}$ Further evidence that cognitive biases can lead physicians to give insufficient weight to their own contributions to antimicrobial resistance is provided in an illuminating recent survey of 889 US physicians, which indicated that they often lacked insight into the broader harms of their own antibiotic prescribing decisions. Most of the respondents expressed concern about the problem of antimicrobial resistance. However, the researchers found that: "While $62 \%$ of respondents agreed that other doctors overprescribe antibiotics, only $13 \%$ agreed that they themselves overprescribe antibiotics". ${ }^{40}$ The researchers concluded that "While most respondents agreed that other doctors overprescribe antibiotics, a much smaller proportion (especially of faculty) felt that they themselves overprescribe". ${ }^{41}$ This significant underestimation by physicians of the contribution that their own antibiotic prescribing decisions are likely having on the broader problems of antimicrobial resistance can be characterised as an example of confirmation bias, where an agent interprets information in a way that confirms a view that they already hold, regardless of whether this information actually supports or undermines that view.

Thus, cognitive biases also seem to be diverting physicians' community-centred dispositions to act in accordance with the virtue of justice from hitting its target, in the context of their antibiotic prescribing decisions. A promising way of addressing this problem is for physicians to develop various 'debiasing' strategies, which can help them carry out what justice requires of them here in more practically intelligent ways. For example, Ian Scott and colleagues suggest that physicians' awareness of their cognitive biases, and their ability to counteract the detrimental effects such biases can have on medical decision-making, can be enhanced by providing them with powerful narratives of patients who have been harmed (e.g. by antimicrobial resistance), and by reflective practice and role modelling. ${ }^{42}$ But while the prescribing decisions of individual physicians are clearly a significant contributor to the problem of antimicrobial resistance, effectively addressing this problem goes well beyond the responsibility of each individual physician to 'smarten up' their own decision-making about antibiotic prescribing by strengthening their medical virtues on their own initiative. For these individual efforts must be complemented by the responsibilities of governments, international organisations, policymakers, and healthcare institutions to create institutional and regulatory environments which are conducive to physicians hitting the targets of the role virtues of medical beneficence and justice. For it can sometimes be difficult for physicians in their antibiotic prescribing behaviour to succeed in hitting the targets of the virtues of medical beneficence and justice, when they are working in contexts where they are frequently

\footnotetext{
${ }^{39}$ See Saposnik et al. (2016), op. cit. Overconfidence and availability bias are the two most frequently researched biases in medical decision making, and they appear to be highly prevalent in this context (though their precise prevalence levels here have not been determined).

${ }^{40}$ Lilian Abbo et al. (2011), p. 715.

${ }^{41}$ Ibid., p. 716.

${ }^{42}$ Ian A Scott et al. (2017). See also Ateev Mehrotra and Jeffrey A. Linder (2017).
} 
confronted with patients' requests for antibiotics, in circumstances where prescribing an antibiotic would be clinically inappropriate.

A good example of such government efforts is the Australian 'Choosing Wisely' initiative, which aims to reduce antibiotic overprescribing (and other forms of poor clinical practice) by providing patients and physicians with guidelines and specific examples of when medications such as antibiotics would be inappropriate. For instance, one such guideline advises against prescribing antibiotics for patients with uncomplicated acute bronchitis. ${ }^{43}$ These guidelines and examples on the Choosing Wisely website encourage discussions between physicians and patients about the appropriateness or otherwise of such medications in the circumstances, and make the boundaries of good medical practice more transparent to patients and doctors. Similarly, the UK Behavioural Insights Team successfully reduced the overprescription of antibiotics by sending letters to GPs in practices with relatively high rates of antibiotic prescription, stating that " $80 \%$ of practices in your local area prescribe fewer antibiotics per head than yours". ${ }^{44}$ Providing these doctors with such benchmarking information helped nudge them to recognise and counteract their biases towards prescribing antibiotics, and thereby helped enable these doctors' medical dispositions to hit their proper targets. ${ }^{45}$

Also, a worthwhile institutional initiative here could be to address authority bias through the creation of 'safe spaces' for junior doctors to be able to anonymously report established practices of poor antibiotic prescribing, without necessarily jeopardising their professional relationships with consultants and senior physicians.

\subsection{Conclusion}

The antibiotic prescribing decisions made by physicians have clearly played a significant role in increasing antimicrobial resistance. Due to their monopoly of expertise in their professional roles, physicians are especially well-placed to be aware of, and to combat, the broader problems which certain sorts of antimicrobial prescribing decisions can result in. Virtuous physicians owe the community a readiness to serve it by taking steps to reduce - and hopefully eliminate altogether - antibiotic prescribing decisions that are contrary to the virtues of medical beneficence and justice. In this chapter, I have outlined some strategies that individual physicians, institutions, and healthcare policymakers can develop to help physicians to hit the targets of those virtues, and to thereby play their part in redressing the problems of antimicrobial resistance.

\footnotetext{
${ }^{43}$ See Choosing Wisely Australia, https://www.choosingwisely.org.au/.

${ }^{44}$ Michael Hallsworth et al. (2016). See also David Halpern (2015).

${ }^{45}$ See also Kiran Iyer (2017), and Fay Niker (2018).
} 


\section{References}

Abbo, Lilian, et al. 2011. Faculty and resident physicians' attitudes, perceptions, and knowledge about antimicrobial use and resistance. Infection Control and Hospital Epidemiology 32 (7): 714-718.

Antibiotic Expert Groups. 2019. Therapeutic guidelines: Antibiotic, version 16. Melbourne, Therapeutic Guidelines. https://tgldcdp.tg.org.au/guideLine?guidelinePage=Antibiotic\&from page $=$ etgcomplete

Aristotle. 1980. The Nicomachean Ethics. Trans. W.D. Ross. Oxford: Oxford University Press.

Australian Commission on Safety and Quality in Health Care. 2020. Antimicrobial prescribing practice in Australian hospitals: Results of the 2018 Hospital National Antimicrobial Prescribing Survey, January 2020. https://www.ncas-australia.org/ncas-publications.

Blumenthal-Barby, J.S., and H. Krieger. 2015. Cognitive biases and heuristics in medical decisionmaking: A critical review. Medical Decision Making 35 (4): 539-557.

Britten, Nicky. 1995. Patients' demands for prescriptions in primary care. British Medical Journal 310 (29): 1084-1085.

Britten, Nicky, and Obioha Ukoumunne. 1997. The influence of patients' hopes of receiving a prescription on doctors' perceptions and the decision to prescribe: A questionnaire survey. British Medical Journal 315 (7121): 1506-1510.

Collignon, Peter J. 2002. Antibiotic resistance. Medical Journal of Australia 177 (6): 325-329.

Costelloe, Céire, et al. 2010. Effect of antibiotic prescribing in primary care on antimicrobial resistance in individual patients: Systematic review and meta-analysis. British Medical Journal 340 (18): c2096.

Crisp, Roger. 2015. The duty to do the best for one's patient. Journal of Medical Ethics 41: 220-223.

Cui, Dan, et al. 2017. Use of and microbial resistance to antibiotics in China: A path to reducing antimicrobial resistance. Journal of International Medical Research 45 (6): 1768-1778.

Dallas, Anthea, et al. 2014. Antibiotic prescribing for the future: Exploring the attitudes of trainees in general practice. British Journal of General Practice 64 (626): e561-e567.

Fleming-Dutra, Katherine E., et al. 2016. Prevalence of inappropriate antibiotic prescriptions among US ambulatory care visits, 2010-2011. Journal of the American Medical Association 315 (17): 1864-1873.

Hallsworth, Michael, et al. 2016. Provision of social norm feedback to high prescribers of antibiotics in general practice: A pragmatic national randomised controlled trial. The Lancet 387 (10029): 1743-1752.

Halpern, David. 2015. Inside the nudge unit. London: WH Allen.

Hursthouse, Rosalind. 1999. On virtue ethics, Oxford: Oxford University Press.

Iyer, Kiran. 2017. Nudging virtue. Southern California Interdisciplinary Law Journal 26: 469-492.

Linder, Jeffrey A., et al. 2014. Time of day and decision to prescribe antibiotics. JAMA Internal Medicine 174 (12): 2029-2031.

Macfarlane, John, et al. 1997. Influence of patients' expectations on antibiotic management of acute lower respiratory tract illness in general practice: Questionnaire study. British Medical Journal 315 (7117): 1211-1214.

McCullough, Amanda R., et al. 2017. Antibiotics for acute respiratory infections in general practice: Comparison of prescribing rates with guideline recommendations. Medical Journal of Australia 207 (2): 65-69.

McMahon, Marilyn. 1992. Dangerousness, confidentiality, and the duty to protect. Australian Psychologist 27 (1): 12-16.

Mehrotra, Ateev, and Jeffrey A. Linder. 2017. Tipping the balance towards fewer antibiotics. JAMA Internal Medicine 176 (11): 1649-1650.

Metlay, Joshua P., et al. 2002. Tensions in antibiotic prescribing: Pitting social concerns against the interests of individual patients. Journal of General Internal Medicine 17: 87-94.

Milgram, Stanley. 2010. Obedience to authority: An experimental view. London: Pinter and Martin. 
Millar, M. 2020. A capability perspective on antibiotic resistance, inequality, and child development. In Ethics and drug resistance: Collective responsibility for global public health. Cham: Springer.

Niker, Fay. 2018. Policy-led virtue cultivation: Can we 'nudge' citizens towards developing virtues? In The theory and practice of virtue education, ed. T. Harrison and D. Walker, 153-168. London: Routledge.

Nussbaum, Martha C. 2006. Frontiers of justice: Disability, nationality, species membership. Cambridge, MA: Harvard University Press.

Oakley, Justin. 1994. Sketch of a virtue ethics approach to health care resource allocation. Monash Bioethics Review 13 (4): 27-33.

2018a. Toward an empirically informed approach to medical virtues. In The Oxford handbook of virtue, ed. Nancy E. Snow, 571-590. Oxford: Oxford University Press.

2018b. Creating regulatory environments for practical wisdom and role virtues in medical practice. In Cultivating moral character and virtue in professional practice, ed. David Carr, 83-95. London: Routledge.

Oakley, Justin, and Dean Cocking. 2001. Virtue ethics and professional roles, Cambridge: Cambridge University Press.

Angus Randall. 2017. Antibiotics: GPs prescribing to patients at up to nine times higher than current guidelines. ABC Radio, The World Today, 11 July 2017. http://www.abc.net.au/ news/2017-07-10/australians-overloaded-with-antibiotics-research/8693600.

Russell, Daniel C. 2009. Practical intelligence and the virtues. New York: Oxford University Press. 2015. What virtue ethics can learn from utilitarianism. In The Cambridge companion to utilitarianism, ed. Ben Eggleston and Dale E. Miller, 258-279. Cambridge: Cambridge University Press.

Saposnik, G., D. Redelmeier, C.C. Ruff, and P.N. Tobler. 2016. Cognitive biases associated with medical decisions: A systematic review. BMC Medical Informatics and Decision Making 16 (138): $1-14$.

Saripanidas, Stavros. 2016. Antibiotic abuse in Greece. British Medical Journal 355: 16328.

Scott, Ian A., et al. 2017. Countering cognitive biases in minimising low value care. Medical Journal of Australia 206 (9): 407-411.

Sharma, Aditya, et al. 2017. Estimating the future burden of multidrug-resistant and extensively drug-resistant tuberculosis in India, the Philippines, Russia, and South Africa: A mathematical modelling study. The Lancet: Infectious Diseases 17 (7): 707-715.

Snow, Nancy E. 2009. How ethical theory can improve practice: Lessons from Abu Ghraib. Ethical Theory and Moral Practice 12 (5): 555-568.

-2010. Virtue as social intelligence: An empirically grounded theory. New York: Routledge.

Swanton, Christine. 2003. Virtue ethics: A pluralistic view. Oxford: Oxford University Press.

Tversky, Amos, and Daniel Kahneman. 1981. The framing of decisions and the psychology of choice. Science 211 (4481): 453-458.

Yao, Zhenjiang, et al. 2015. Healthcare associated infections of Methicillin-resistant Staphylococcus aureus: A case-control-control study. PLoS One 10 (10): e0140604. 
Open Access This chapter is licensed under the terms of the Creative Commons Attribution 4.0 International License (http://creativecommons.org/licenses/by/4.0/), which permits use, sharing, adaptation, distribution and reproduction in any medium or format, as long as you give appropriate credit to the original author(s) and the source, provide a link to the Creative Commons licence and indicate if changes were made.

The images or other third party material in this chapter are included in the chapter's Creative Commons licence, unless indicated otherwise in a credit line to the material. If material is not included in the chapter's Creative Commons licence and your intended use is not permitted by statutory regulation or exceeds the permitted use, you will need to obtain permission directly from the copyright holder. 